\title{
Development of an Interactive Building Energy Design Software Tool
}

\author{
Niccolò Aste, Rajendra Singh Adhikari, Claudio Del Pero \\ and Fabrizio Leonforte
}

\begin{abstract}
Energy efficiency is one of the most important topics worldwide, nevertheless, each region has its own specifics, which have to be considered. This is especially important for online tools that deliver comprehensive information on energy efficiency and renewable energy for average consumer as well as for municipalities and governments. These tools are essential for energy retrofit of existing building for energy saving as well as designing of new energy-efficient buildings and also for designing and sizing renewable energy technologies such as solar home system. A dynamic interactive building energy design software tool has been developed especially tailored taking care about main features required for Sub-Saharan African context, under a joint agreement (research project) between Politecnico di Milano and UN-Habitat. In this chapter, the results of testing of the software tool for building geometry have been presented.
\end{abstract}

Keywords Building energy efficiency $\cdot$ Design tool $\cdot$ BESTenergy $\cdot$ Testing

\section{Introduction}

Energy used in commercial and residential buildings accounts for a significant percentage of the total national energy consumption. It is estimated that $40 \%$ of the total electricity generated in the region is used in buildings alone, consuming more energy than the transport and industry sectors. The building sector encompasses a diverse set of end-use activities, which have different energy use implications. The amount of energy used for cooling, heating and lighting is directly related to the building design, building materials, the occupants' needs and behavior and the surrounding microclimate. Majority of modern buildings in Sub-Saharan Africa (mainly tropical climates) are replicas of buildings designed for the western world (cold and temperate climates) and do not take into consideration the differences in climate. As a result, buildings are heavily reliant on artificial means for indoor comfort, i.e., cooling,

N. Aste $\cdot$ R. S. Adhikari $(\bowtie) \cdot$ C. Del Pero $\cdot$ F. Leonforte

Architecture, Built Environment and Construction Engineering-ABC Department, Politecnico di Milano, Milan, Italy

e-mail: rajendra.adhikari@polimi.it 
heating and lighting. The problem is that inefficient design and construction using inadequate materials, combined with the poor understanding of thermal comfort, passive building principles and energy conscious behavior, have led to tremendous energy wastage.

Therefore, energy efficiency is one of the most important topics worldwide, nevertheless, each region has its own specifics, which have to be considered. This is especially important for online tools that deliver comprehensive information on energy efficiency and renewable energy for average consumer as well as for municipalities and governments. These tools are essential for energy retrofit of existing building for energy saving as well as designing of new energy-efficient buildings and also for designing and sizing renewable energy technologies such as solar home system. Addressing these issues, the UN-Habitat programme "Promoting Urban Energy for climate change in developing countries" has the objective to mainstream energy efficiency and renewable energy measures in the delivery of sustainable and friendly built environment.

\section{Interactive Building Energy Design Software Tool}

Nowadays' need to meet sustainability requirements, limit environmental impacts and reduce operating costs imposes a structural modification of the architectural design process (Fig. 1), calling for an integrated approach in building energy performance analysis and planning, from the very preliminary design phase. In this framework, an accurate estimation of buildings' energy consumptions, related to the different uses and needs, becomes an essential design and evaluation tool.

At present, the most widespread methods to evaluate building energy performance rely on simplified steady-state calculations based on average reference data. Such methods are quite obsolete and inaccurate, as they do not properly account for the complexity of phenomena impacting energy performance (thermal inertia, temporal
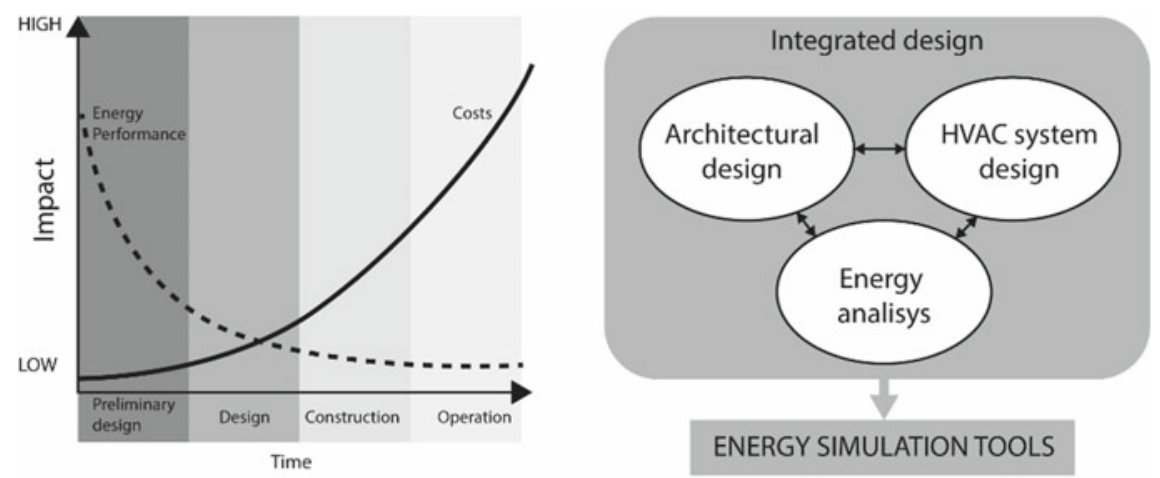

Fig. 1 Impact of energy expertise integration and integrated design approach 
fluctuations, peaks and dips of boundary conditions) and prove to be particularly inadequate in the case of tropical climates and cooling-dominated buildings. In these scenarios, characterized by dynamic paths affecting building thermal behavior and energy performance, designers need more accurate, hourly based methods. These methods, which take into consideration the actual building's thermal response (heat absorption and release induced by the variation of outdoor conditions at different times of the day) are named dynamic state calculations.

Dynamic state calculations are processed by simulation engines and, due to their level of sophistication, are usually difficult to run, especially when they are not provided with a graphical user interface.

In order to enforce an integrated design approach inclusive of energy performance aspects, adequate tools must be developed, able both to correctly encompass the complex interactions between energy flows and design choices and to be user-friendly.

To overcome the above difficulties, Politecnico di Milano in collaboration with UN-Habitat developed an open-source, free-redistributable, intuitive and powerful building energy simulation software, especially tailored taking care about main features required in the African context.

This energy tool provides the following main characteristics:

- Reliable dynamic building energy simulation that is the only method which ensures a correct evaluation in cooling-dominated buildings and in tropical climates, where dynamic paths heavily affect buildings' thermal behavior and energy performance, in which designers need accurate, hourly based methods, considering the actual building thermal response and its heat absorption and release due to the variation of the outdoor conditions throughout the day. This is performed by EnergyPlus simulation engine, which is the state of the art in this item.

- The hardness in compiling a building energy model is avoided by an intuitive interface based on SketchUp, coupling, in this way, the easiness in geometric modeling of the modeling tool with the reliability and accuracy of EnergyPlus. In fact, users can build an energy model by using SketchUp drawing tools and dedicated interfaces, so that the plugin creates the input data file to be processed by the simulation engine with few easy steps. Also, some macros are developed to generate geometries and to automate modeling process.

In such respect, the so-called BESTenergy was born. A conceptual scheme of the software is shown in Fig. 2. BESTenergy includes the state-of-the-art knowledge in building energy estimation and at the same time supports architectural choices. Through this platform, users can keep track in an interactive way of all the consequences emerging from building energy behavior in relation to design choices by considering the overall energy performance-simultaneously including heating, cooling and lighting demands.

More in detail, BESTenergy takes advantage of a SketchUp plugin to run dynamic state building energy simulations using the EnergyPlus (Crawley et al. 2001) simulation engine, thus coupling the handiness of SketchUp geometric modeling with the reliability and accuracy of EnergyPlus (Crawley et al. 2005). 


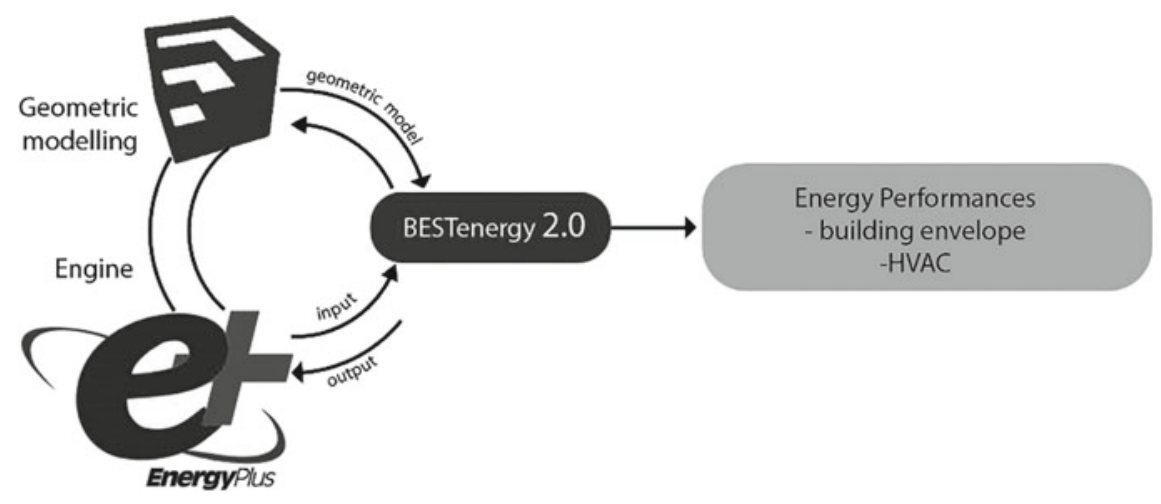

Fig. 2 BESTenergy software conceptual scheme

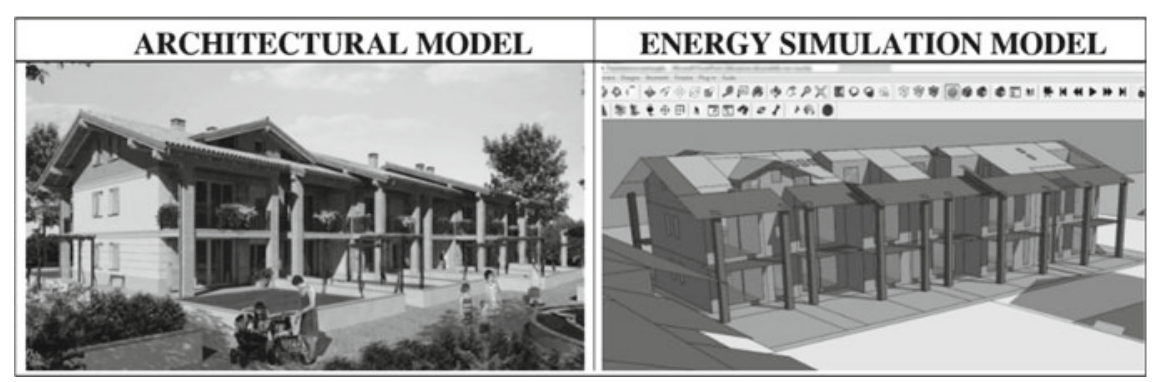

Fig. 3 Comparison between the architectural model and the simulation model in BESTenergy

Thanks to BESTenergy, users can build an energy model exploiting SketchUp drawing tools and dedicated interfaces, while the plugin tool creates an input data file to be processed by the simulation engine. A comparison between a real architectural model and energy simulation model has been shown in Fig. 3.

\section{BESTenergy Simulation Platform}

BESTenergy allows to comprehensively optimize energy resources in the building design phase by looking for the best compromise among different energy services. It also proves to be particularly suitable for evaluating real performances of an already designed (or in progress) building, providing accurate estimates of its features under operating conditions.

In particular, BESTenergy can evaluate

- heating, cooling and lighting energy demands on the same model and their interaction, letting the user to interactively optimize overall energy behavior; 
- effects of movable shading devices;

- thermal comfort levels both by using adaptive comfort models and more traditional ones;

- natural ventilation effects, coupling an energy model with a ventilation model, which is usually split into different evaluation tools;

- local evaluations, as behaviors of each building surface, as its temperature, its amount of heat gain and so on;

- daylighting and simple lighting systems;

- hourly power demand;

- HVAC systems sizing;

- HVAC system hourly operation and response.

Because of its relevance in warm environments (African context), a special effort was dedicated to developing interfaces to model natural ventilation effects: The EnergyPlus airflow network mass transfer model included in BESTenergy enables users to calculate at each time step the actual ventilation flow taking into account the openings' geometry, their relative positions and opening time intervals. These evaluations are directly coupled to the overall building thermal model, and therefore, they interactively affect simulation outputs, avoiding a separate evaluation based on other specific tools.

During its development stages, BESTenergy was tested in university courses, professional trainings and in the context of research studies and professional consultations (Fig. 4), allowing to gather a sample of users characterized by a quite broad range of knowledge backgrounds. As a result, significant on-field feedbacks emerged that proved useful in understanding how to improve the platform's friendliness and functionalities.
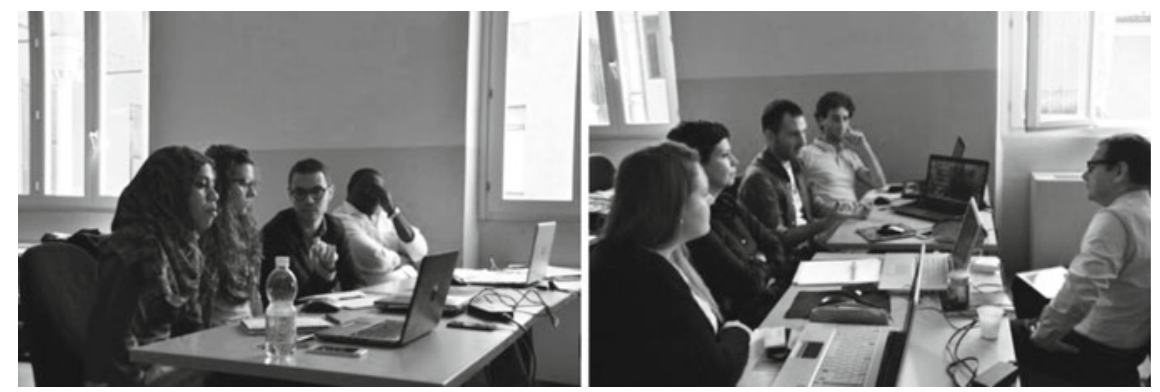

Fig. 4 Students and tutors dealing with BESTenergy during a workshop 


\section{Testing Building Geometry Generated by the BESTenergy Tool}

New tools must be tested to verify the validity of results of their use and to identify possible peculiarities and qualifications of how they generate those results. To verify building geometry modeled by BESTenergy, the research team selected the design of a three-story hypothetical office building (Fig. 5) that features non-trivial geometry configuration and has been previously repetitively used in the testing of other new simulation tools and utilities. It is known as the BLIS Test Building, developed jointly by the Interoperability Team at the Environmental Energy Technologies Division of the Lawrence Berkeley National Laboratory and Digital Alchemy.

The total net test building area is $1639.82 \mathrm{~m}^{2}$ and gross roof area is $574.63 \mathrm{~m}^{2}$ with an expected building occupancy between 90 and 100 people.

The wireframe of the building geometry model generated by BESTenergy is shown (Fig. 6); since it was drawn with SketchUp, it is a "center-line" model (walls' and slabs' thickness is not shown) which defines area and volume values that are larger than they really are. For comparison with the geometry generated by BESTenergy, the wireframe model of the test building geometry that is a full 3D CAD geometry model transformed for use in EnergyPlus is also shown (right).

Testing of the BESTenergy tool involved four steps: (1) drawing building geometry of the BLIS test building with SketchUp and applying BESTenergy as the interface to create a geometry compatible with EnergyPlus input requirements; (2) recording EnergyPlus geometry output data resulting from the simulation with BESTenergy; (3) executing the EnergyPlus Input Definition File (IDF), generated by the BESTenergy run, in independent "stand-alone" EnergyPlus runs; (4) comparing the reported detailed building geometry information to the original geometry information for the

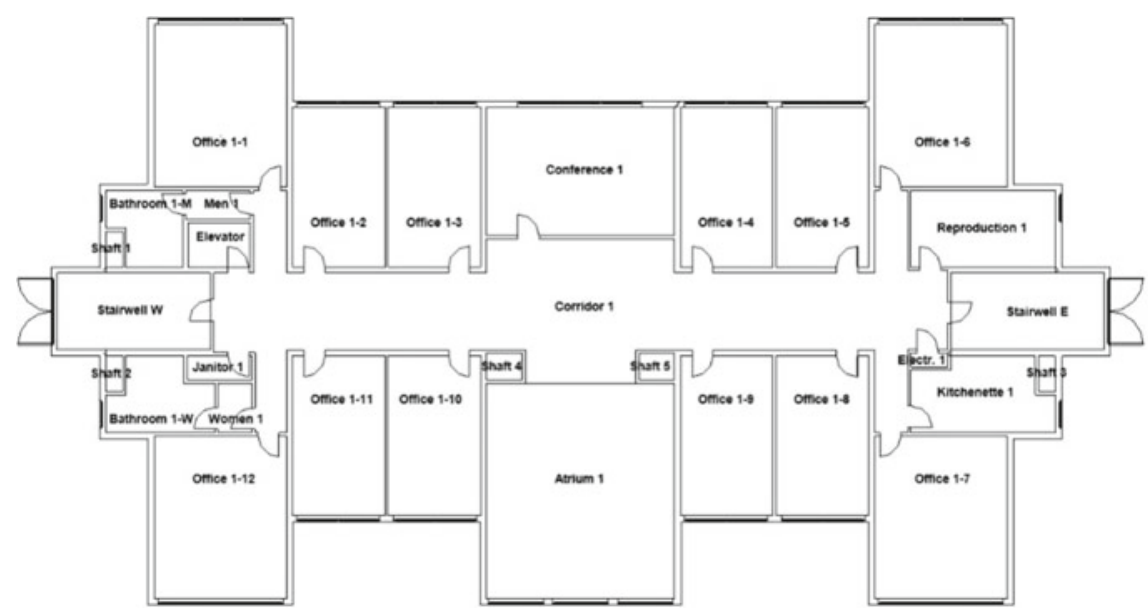

Fig. 5 Typical floor of the test building 


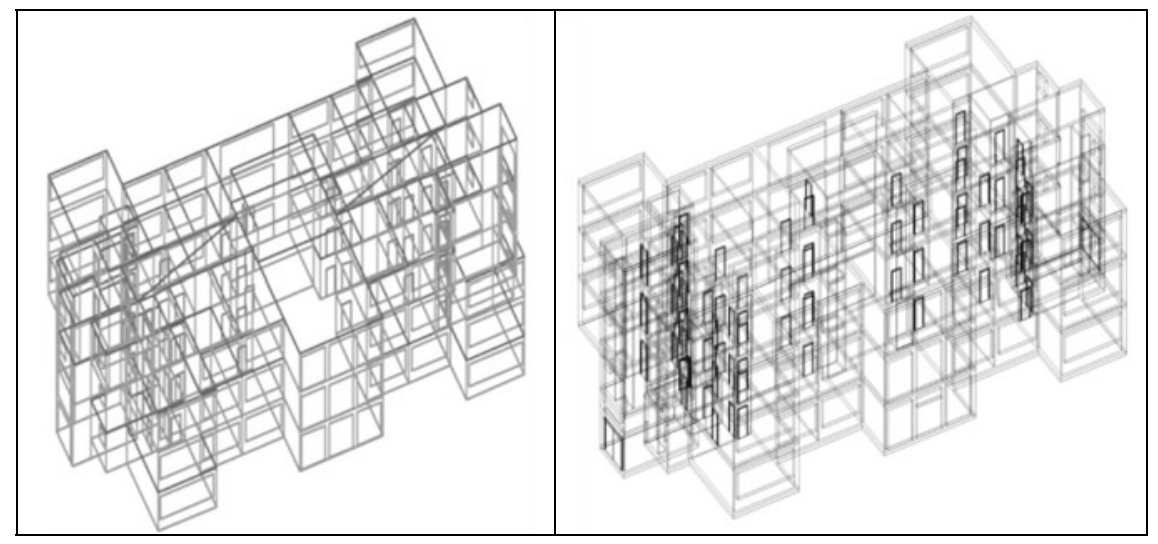

Fig. 6 Wireframe image of the test building model geometry generated with BESTenergy and imported into EnergyPlus (left) and wireframe image of the original test building geometry model generated in Revit 2018, then transformed and imported into EnergyPlus (right)

BLIS building that had been generated by Revit and subsequently semiautomatically transformed and run by EnergyPlus.

All EnergyPlus runs related to the testing of the BESTenergy tool and used in the comparison were executed with the EnergyPlus version 8.9 simulation engine (which currently is the latest EnergyPlus release). The original geometry of the BLIS test building was first defined in ArchiCAD (Graphisoft 2018)_and subsequently also in Revit (Autodesk 2018) — and then extended with space boundary definitions automatically calculated by SBT and verified with the Solibri Model Checker (Solibri 2018). Modelers involved in the testing and verification of the BESTenergy tool are experienced in building geometry modelers with a long record of involvement with BEP simulation.

First of all, the net floor areas for each space/zone in the test building (called "BESTenergy Area"), calculated by EnergyPlus using the geometry model generated by the BESTenergy tool, have been compared with net floor areas of the original geometry model generated by Revit. After that, a similar evaluation was carried out on the volume. A sample of the obtained data is reported in Table 1.

After that the "Difference in Area" that represents difference between the same space/zone area values calculated from the BESTenergy geometry model and the original BLIS building geometry model has been evaluated. In each case, the difference is expressed as percentage of the smaller value in the pair. Calculated differences vary widely: The smallest difference is $3.06 \%$; the largest is $18.25 \%$. This difference is caused by the center-line model and cannot be standardized, as it depends on the actual thickness of every building element (wall or slab) that is represented in that center-line geometry model.

In fact, EnergyPlus geometry model is a surface geometry model. It accepts building surface information as defined in its input file and calculates itself all other nonsurface information it may need from available surface data (such as space/zone 
Table 1 Quantitative comparison of building geometries in test

\begin{tabular}{|c|c|c|c|c|c|c|}
\hline Space/zone name & On floor(s) & $\begin{array}{c}\text { BESTenergy } \\
\text { area }\left(\mathrm{m}^{2}\right)\end{array}$ & $\begin{array}{c}\text { Test building } \\
\text { area } \\
\left(\mathrm{m}^{2}\right)\end{array}$ & $\begin{array}{c}\text { Difference } \\
\text { in } \\
\text { area }(\%)\end{array}$ & \begin{tabular}{|c|}
$\begin{array}{c}\text { BESTenergy } \\
\text { volume } \\
\left(\mathrm{m}^{3}\right)\end{array}$ \\
\end{tabular} & $\begin{array}{c}\begin{array}{c}\text { Test building } \\
\text { volume } \\
\left(\mathrm{m}^{3}\right)\end{array} \\
\end{array}$ \\
\hline OFFICE $1-1$ & 1 & 28.99 & 28.07 & 3.28 & 92.76 & 85.33 \\
\hline OFFICE $1-2$ & 1 & 20.67 & 19.35 & 6.82 & 66.16 & 58.82 \\
\hline OFFICE $1-3$ & 1 & 20.62 & 19.29 & 6.89 & 65.97 & 58.64 \\
\hline OFFICE $1-4$ & 1 & 20.67 & 19.29 & 7.15 & 66.16 & 58.64 \\
\hline OFFICE 1.5 & 1 & 20.61 & 19.35 & 6.51 & 65.97 & 58.82 \\
\hline OFFICE $1-6$ & 1 & 28.99 & 28.07 & 3.28 & 92.76 & 85.33 \\
\hline ELEVATOR & $1,2,3,4$ & 4.25 & 3.62 & 17.40 & 52.33 & 45.90 \\
\hline CONFERENCE 1 & 1 & 32.90 & 31.48 & 4.51 & 105.28 & 95.70 \\
\hline REPRODUCTION 1 & 1 & 15.60 & 14.55 & 7.22 & 48.67 & 44.23 \\
\hline MEN 1 & 1 & 2.77 & 2.45 & 13.06 & 8.87 & 7.45 \\
\hline BATHROOM 1-M & 1 & 7.60 & 6.71 & 13.26 & 24.31 & 20.40 \\
\hline SHAFT 1 & $1,2,3$ & 0.87 & 0.79 & 10.13 & 8.18 & 7.42 \\
\hline OFFICE $1-7$ & 1 & 28.99 & 28.07 & 3.28 & 92.76 & 85.33 \\
\hline OFFICE $1-8$ & 1 & 20.61 & 19.35 & 6.51 & 65.97 & 58.82 \\
\hline OFFICE $1-9$ & 1 & 20.67 & 19.29 & 7.15 & 66.16 & 58.64 \\
\hline OFFICE $1-10$ & 1 & 20.62 & 19.29 & 6.89 & 65.97 & 58.64 \\
\hline OFFICE $1-11$ & 1 & 20.67 & 19.35 & 6.82 & 66.16 & 58.82 \\
\hline OFFICE $1-12$ & 1 & 28.99 & 28.07 & 3.28 & 92.76 & 85.33 \\
\hline CORRIDOR 1 & 1 & 107.80 & 101.45 & 6.26 & 344.95 & 308.41 \\
\hline ATRIUM 1 & 1 & 55.30 & 52.83 & 4.68 & 176.96 & 169.06 \\
\hline KITCHENETTE 1 & 1 & 13.60 & 12.75 & 6.67 & 43.53 & 38.76 \\
\hline WOMEN 1 & 1 & 2.72 & 2.17 & 25.35 & 8.71 & 6.60 \\
\hline BATHROOM 1-W & 1 & 9.31 & 8.82 & 5.56 & 29.8 & 26.81 \\
\hline JANITOR 1 & 1 & 2.42 & 2.07 & 16.91 & 7.73 & 6.29 \\
\hline ELECTRICAL CLOSET 1 & 1 & 0.70 & 0.67 & 4.48 & 2.25 & 2.04 \\
\hline SHAFT 1 & $1,2,3$ & 0.87 & 0.79 & 10.13 & 8.36 & 7.42 \\
\hline SHAFT 2 & $1,2,3$ & 0.87 & 0.79 & 10.13 & 8.18 & 7.42 \\
\hline SHAFT 3 & $1,2,3$ & 0.87 & 0.79 & 10.13 & 8.18 & 7.42 \\
\hline SHAFT 4 & $1,2,3$ & 1.49 & 1.26 & 18.25 & 13.96 & 11.83 \\
\hline SHAFT 5 & $1,2,3$ & 1.42 & 1.26 & 18.25 & 13.29 & 11.83 \\
\hline Average conditioned & 3 & 26.92 & 25.50 & 5.57 & \multicolumn{2}{|c|}{$\Sigma$ (areas)/no. of conditioned zones } \\
\hline Average ventilated & 3 & 8.46 & 7.77 & 8.89 & \multicolumn{2}{|c|}{$\Sigma$ (areas)/ no. of ventlated zones } \\
\hline
\end{tabular}

volumes), even if such information is available in the input file. Because of that, any comparison of volume data does not add new information, as any volume value is a function of the associated floor area value and is recalculated by EnergyPlus.

Definition of building geometry with "center-line" tools inevitably causes quantitative errors if compared with the real 3D geometry of the given building. Correct geometry can be manually calculated from "center-line" geometry, as illustrated Fig. 7.

Yet, "center-line" CAD tools are popular. They are easier to learn and use than object-oriented model-based CAD tools, require less effort and modeling time, and the modeling software is usually much less expensive. SketchUp is a good example: It is the recommended geometry definition software for OpenStudio applications; it is particularly often used by novices as the graphical user interface (GUI) to building energy performance (BEP) simulation with EnergyPlus that directly defines building 


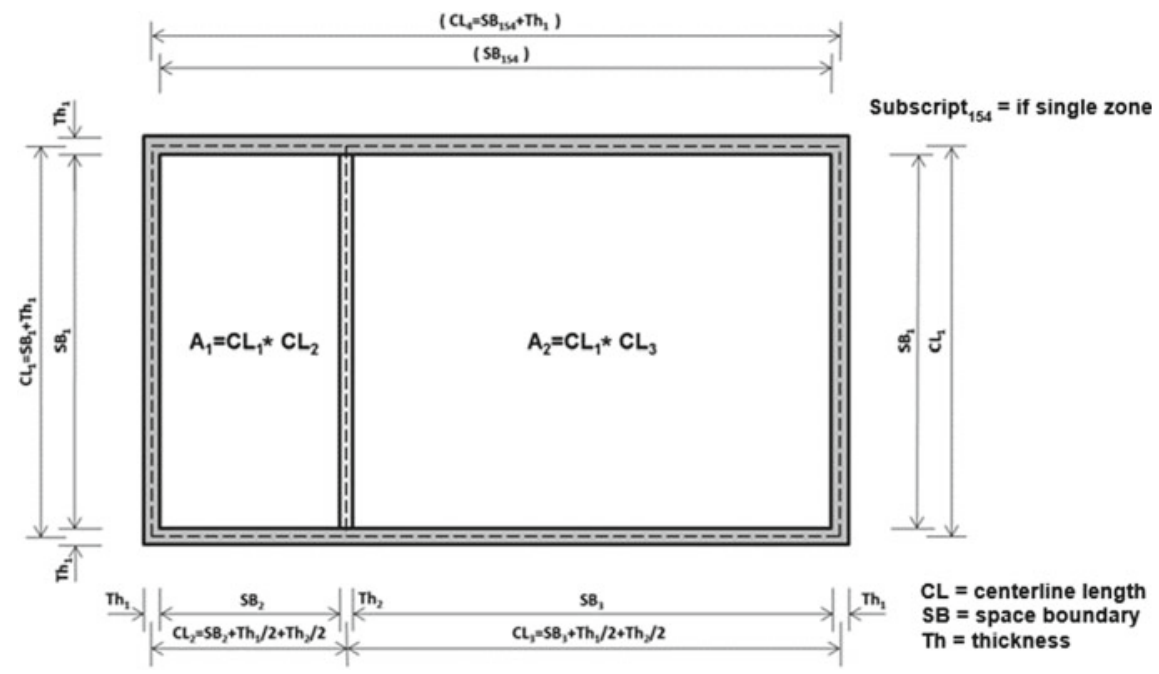

Fig. 7 Example of calculating correct floor area from "center-line" geometry definition (Bazjanac et al. 2016)

geometry for the simulation even though the defined geometry is only an approximation.

The critical question then is what is acceptable engineering error in BEP modeling and simulation? The International Standards Organization (ISO 2018) and other standardization bodies offer no help in defining acceptable error in BEP modeling and simulation. Search of the Internet reveals a lively discussion of the subject within the buildings industry and an apparent consensus among its participants: The acceptability of a percent error depends on the application. Buildings industry consensus for acceptable errors in numerical results appears to be $5 \%$ or less.

Perhaps the critical question above should be refined: What is acceptable accuracy, precision or error that makes a given building geometry model still useful? If one must use data showing high error values, one should (1) understand the cause(s) of error(s); (2) assess the significance and impact of error(s) in question and (3) if available/possible, compare calculations/predictions to measured values, then calibrate the model accordingly.

In conclusion, BESTenergy can be considered a reliable tool to use for energy evaluation, especially when overestimation is not harmful and results are informative and useful. Overall, "center-line" error depends on the sum of ratios of all object thicknesses, not on the lengths or heights of the corresponding walls and slabs. It often significantly exceeds 5\%. Building geometry defined by SketchUp, and consequently, used by the BESTenergy tool, is an approximation of the true geometry of the building being modeled; using it in EnergyPlus will overestimate building's thermal loads and will likely result in oversized HVAC systems selected for the building. Yet, by using the BESTenergy tool as a GUI to prepare input for EnergyPlus, modeling building 
geometry is significantly less challenging and less time consuming than developing full CAD-type geometry models.

The differences between BESTenergy and the original BLIS building geometry models can be directly attributed to the difference in the types of geometry modeling involved. For the test building geometry used in the BESTenergy generated geometry model evaluation, the differences exceed the apparent commonly acceptable industry error tolerance of $5.00 \%$. The "center-line" modeling approach leaves out the thickness of the modeled objects (walls or slabs); this inevitably results in the definition of larger areas and volumes than those from modeled by object-oriented CAD.

Modeling building geometry for use in EnergyPlus requires a certain level of precision. The quality of modeling and BEP simulation depends directly on the modeler's ability and provided effort. Novice modelers and modelers with little previous exposure to modeling knowledge will find BESTenergy to be a most useful tool which quickly advances their modeling knowledge and skills.

Value and utility of BEP modeling and simulation are often directly proportional to the skill of the modeler. Successful BEP modeling and simulation require several skills: (1) knowledge and understanding of BEP simulation; (2) working knowledge of model-based CAD and other simulation-related software tools; (3) good understanding of building physics; (4) understanding of building systems and HVAC and (5) ability to effectively engage in large database analysis. Successful modeling is a collaborative process involving, besides the modeler(s), the entire building design team, project management and consultants, as well as relevant government officials. The buildings industry would greatly benefit from a larger number of skilled modelers than available in the currents pool. Modeling is not a skill acquired at birth; it is a skill that is learned and requires experience. For that, future modelers need access to properly designed, funded and executed educational programs, as well as timely opportunities and patience to practice newly acquired and developed skills.

\section{References}

Autodesk (2018) Revit Architecture 2018. https://www.autodesk.com/products/revit/architecture

Bazjanac V, Maile T, Nytsch-Geusen C (2016) Generation of building geometry for energy performance simulation using Modelica. In: Proceedings of the CESBP/BauSIM 2016 conference, Dresden, September 2016

Crawley DB, Lawrie LK, Winkelmann FC, Pedersen CO (2001) EnergyPlus: a new-generation building energy simulation program. In: Proceedings of forum 2001: solar energy: the power to choose, Washington, DC, April 2001. ASES, Boulder, Colorado

Crawley DB, Hand JW, Kummert M, Griffith BT (2005) Contrasting the capabilities of building energy performance simulation programs. In: Proceedings of building simulation 2005, Montreal Graphisoft (2018) ArchiCAD 2022. https://www.graphisoft.com/archicad/

Solibri (2018) Solibri model checker. https://www.solibri.com/howit-works 
Open Access This chapter is licensed under the terms of the Creative Commons Attribution 4.0 International License (http://creativecommons.org/licenses/by/4.0/), which permits use, sharing, adaptation, distribution and reproduction in any medium or format, as long as you give appropriate credit to the original author(s) and the source, provide a link to the Creative Commons license and indicate if changes were made.

The images or other third party material in this chapter are included in the chapter's Creative Commons license, unless indicated otherwise in a credit line to the material. If material is not included in the chapter's Creative Commons license and your intended use is not permitted by statutory regulation or exceeds the permitted use, you will need to obtain permission directly from the copyright holder.

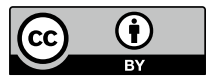

